\title{
Simulation of Forward Propagated Signals in Acoustic Time Reversal
}

\section{Tommiska, Oskari Mikael}

IEEE

2019

Tommiska, O M , Moilanen , P , Mustonen , J A , Rauhala , T , Gritsevich , M , Salmi , A \& Haeggström , E 2019 , Simulation of Forward Propagated Signals in Acoustic Time Reversal . in 2019 IEEE International Ultrasonics Symposium (IUS) . IEEE International Ultrasonics Symposium , IEEE , pp. 2515-2518 , IEEE International Ultrasonics Symposium , Glasgow , United Kingdom , 06/10/2019 . https://doi.org/10.1109/ULTSYM.2019.8926189

http://hdl.handle.net/10138/326120

https://doi.org/10.1109/ULTSYM.2019.8926189

acceptedVersion

Downloaded from Helda, University of Helsinki institutional repository.

This is an electronic reprint of the original article.

This reprint may differ from the original in pagination and typographic detail.

Please cite the original version. 


\section{Simulation of Forward Propagated Signals in Acoustic Time Reversal}

\author{
Oskari Tommiska \\ Electronics lab., Physics dept. \\ Univ. of Helsinki \\ Helsinki, Finland
}

\author{
Maria Gritsevich \\ Electronics lab., Physics dept. \\ Univ. of Helsinki \\ Helsinki, Finland
}

\author{
Petro Moilanen \\ Altum Technologies \\ Finland
}

Finland

\author{
Joonas Mustonen \\ Electronics lab., Physics dept. \\ Univ. of Helsinki \\ Helsinki, Finland
}

Ari Salmi
Electronics lab., Physics dept.
Univ. of Helsinki
Helsinki, Finland

\begin{abstract}
Time reversal (TR) is a technique based on the reciprocity principle of the wave equation that can be used to focus waves. In an enclosed system, comprising homogenous medium that includes no reflecting targets, it normally is impossible to use time reversal to focus ultrasound into the medium. Our proposed solution to this problem is to use simulations to produce signals that are subsequently time reversed and used for focusing. We show that one can model the forward propagation with finiteelement method (FEM), then time-reverse it, and finally transmit it back to generate a time reversed focus in an experimental setup. The results show that accurate $T R$ focusing can be achieved using simulated signals both in homogenous media and in media including distinct inclusions.
\end{abstract}

Keywords - time reversal, finite element method, simulation

\section{INTRODUCTION}

Time reversal (TR) can be used to focus acoustic waves [1]. The method is based on the reciprocity principle of the wave equation: the initial condition of a known solution can be reached with reversed time [2].

The advantage of the TR method when compared to other focusing methods is that the exact geometry or properties of propagation medium are not needed to perform TR focusing [3]. Applications of TR method are numerous: in seismology [4], in structural health monitoring [5], and in medicine [6].

In principle, a TR focusing experiment employs the following steps: 1) The acoustic signal is actuated at the predefined target point 2) The forward propagation is recorded using transducers surrounding the target. 3 ) The recorded signals are reversed in time and then driven back using the same transducers that were used for recording. As a result, the timereversed backward propagation forms a focal point at the predefined target point. [7].

Target point actuation is typically either a real actuation done using an acoustic source [8] or an echo reflected from an object at the target location [9]. When focusing into an arbitrary location inside an enclosed container, where acoustic source cannot be inserted and no suitable internal structure exists to produce the echoes, performing the actuation becomes problematic. Our solution to this problem is to simulate the target point actuation and the forward propagation. Simulated and time reversed signals can then be used to focus waves in a related real-life geometry.

\section{METHODS}

Simulations were done using COMSOL Multiphysics 5.4 finite element method (FEM) simulation software.

The modelled experimental setup consists of an acrylic water tank with four Langevin piezoelectric transducers attached to it. The setup was modelled in 3D, including the four working, accurately modelled transducers (Fig. 1).

To reduce the size of the simulation geometry, only a small portion of the tank was modelled. This was assumed to be a valid approximation, since the contributions of reflections from the top and bottom of the container were assumed to be small. In the Experimental Validation -section this approximation is showed to be valid. The top and the bottom boundaries were modelled using low reflecting boundary conditions.

Two different versions of the same basic geometry were simulated: one with only water inside the container and one with three water filled steel pipes residing inside the water domain.

The propagation of acoustic waves in water was simulated using COMSOL's Pressure Acoustics, Transient interface, included in Acoustics module. Solid Mechanics and Electrostatics interfaces were used for modelling the wave propagation in solids and the piezoelectric effects in transducers. All multiphysics couplings were done using COMSOL's builtin multiphysics interfaces. 


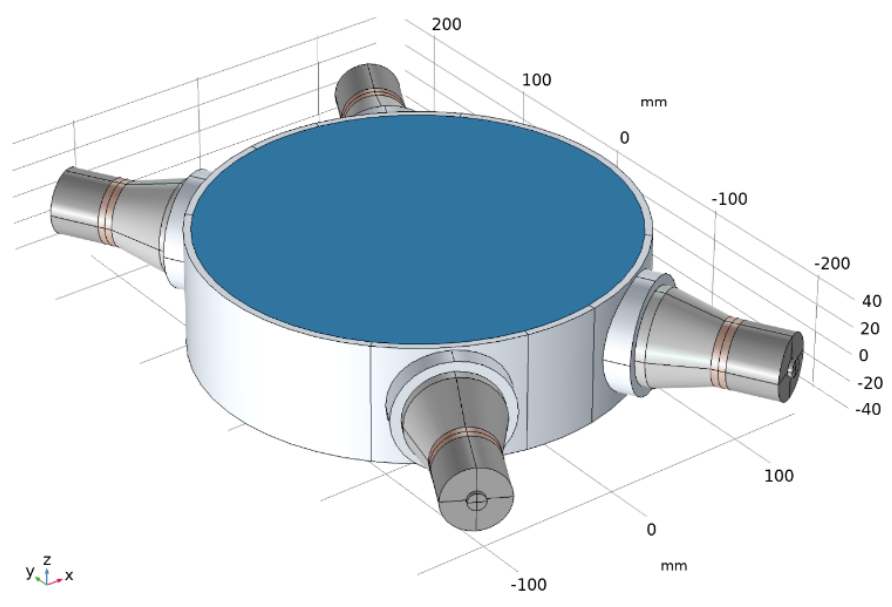

Fig. 1. The simulation geometry consists of a water filled slice of the acrylic container and Langevin transducers.

The forward propagated signal was generated in the simulation by introducing a small, spherical pressure source into the water domain. A short $20 \mathrm{kHz}$ pressure signal was used to produce the forward propagated waves. Simulations were done in the time domain. The propagated wave was recorded as the received electric signals from the simulated transducers. The recorded signals were then exported from COMSOL and used in the experimental setup.

To validate that the TR focusing can be achieved with merely four transducers, also the backwards propagation of the time reversed signals was simulated. In backwards propagation simulations, the simulated transducers were driven with the time reversed versions of the previously recorded simulated signals. The time reversing of the signals was performed in COMSOL using the withsol-function.

\section{SimULATION RESULTS}

Simulation of the forward propagating wave was done in the time domain, with each simulated propagation lasting for $2 \mathrm{~ms}$. Fig. 2 shows the forward propagation of the acoustic wave in the plane holding the transducers.

The electric signals recorded in the cases presented in Fig. 2 are shown in Fig. 3. The backward propagation was simulated by reversing the signals recorded in the forward propagation simulation and by sending them back with the simulated transducers. As a result, propagating waves formed a focal point at the same location where the initial excitation was done (Fig. 4). Since only four transducers were used in TR, several additional, relatively strong side focuses appear. The strongest focus forms at the predefined focal point.

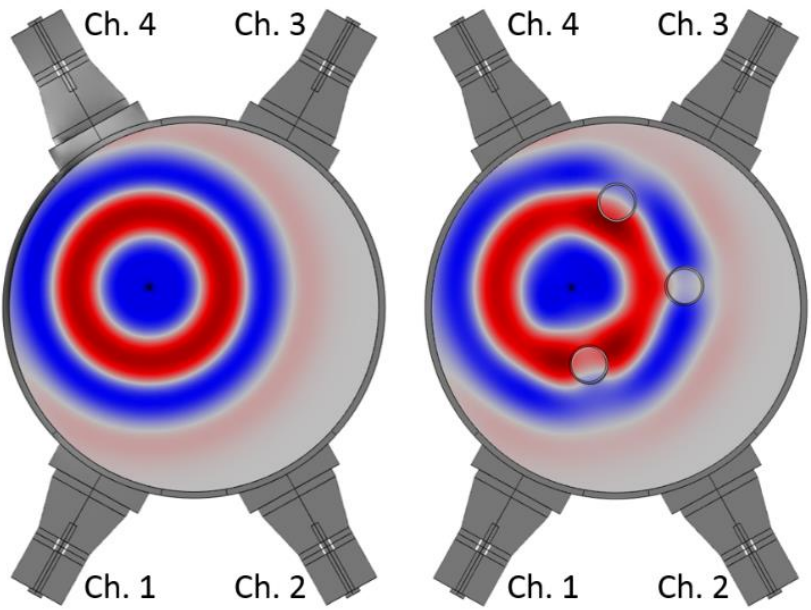

Fig. 2. Examples of time domain simulations of forward propagated signals. Container filled only with water (left), the same container with three added steel pipes (right).
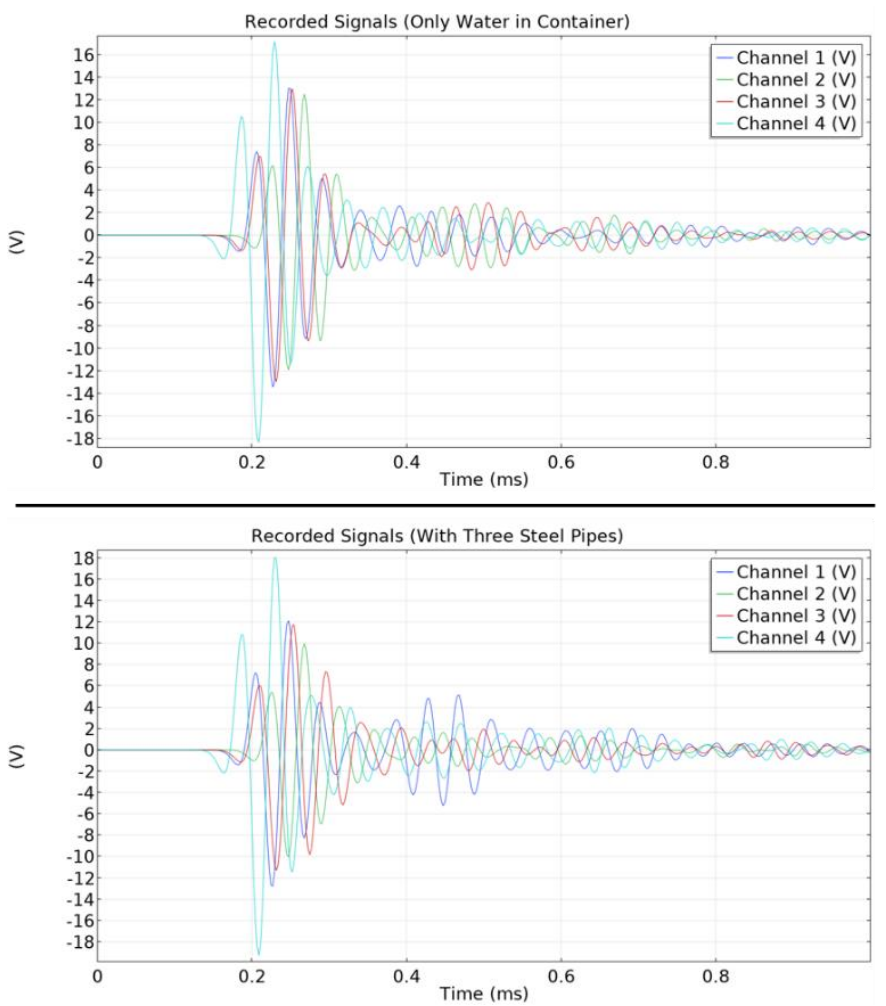

Fig. 3. Examples of recorded electric signals in two cases showed in Fig. 2. 


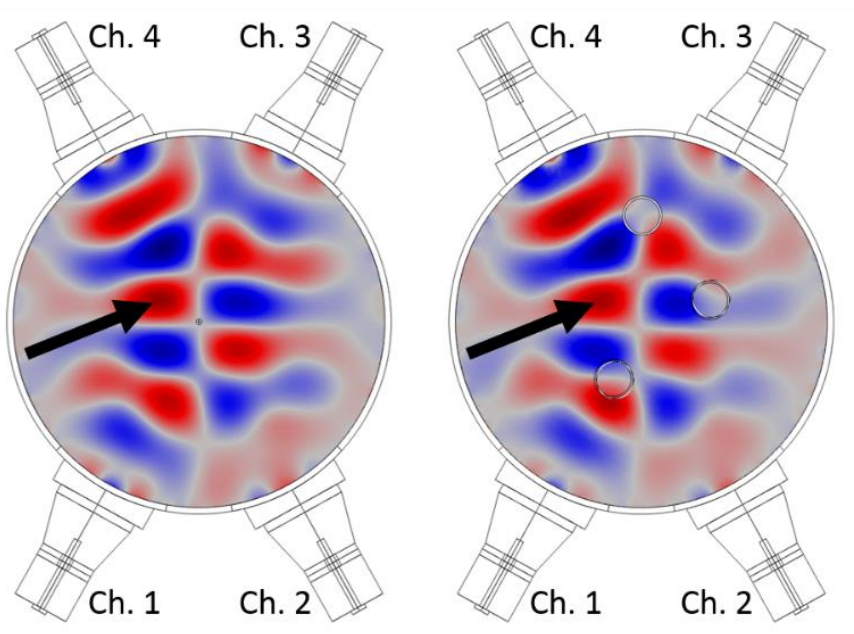

Fig. 4. Time-reversed focusing of waves. The figure shows the moment when the focus forms in time domain simulation. A focus is seen at the point of the initial excitation (black arrow). Container filled only with water (left), and the same container with three added steel pipes (right).

\section{EXPERIMENTAL VALIDATION}

To validate the simulation model, pressure scans were performed during TR focusing in the experimental setup.

For the cases showed in Fig. 2-4, two lines were scanned in each case: one parallel to the $\mathrm{x}$-axis and one parallel to the $\mathrm{y}$ axis (Fig. 5-6). These lines were chosen so that they intersected at the focal point. The acoustic pressure was measured using an omnidirectional hydrophone (Brüel \& Kjær type 8103). In the case with added pipes (Fig. 6), line scans were shorter to avoid colliding the hydrophone with the pipes.
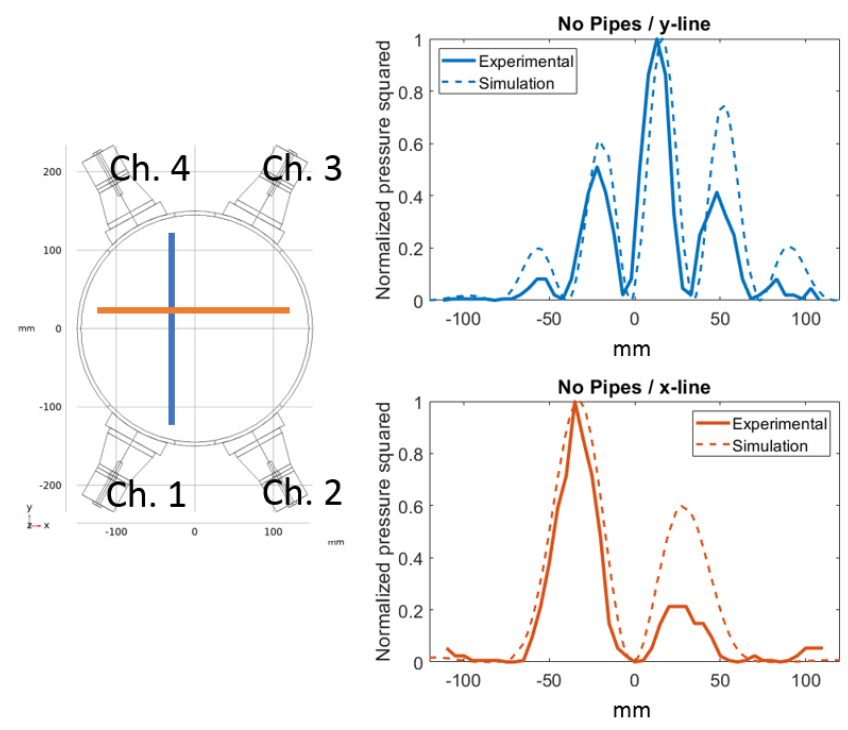

Fig. 5. Validation of the simulation without internal structure. Colored lines in the picture (left) show the line scans. The focal point is at the intersection of the lines. Graphs show comparisons of experimental measurement (solid line) and simulation (dashed line).

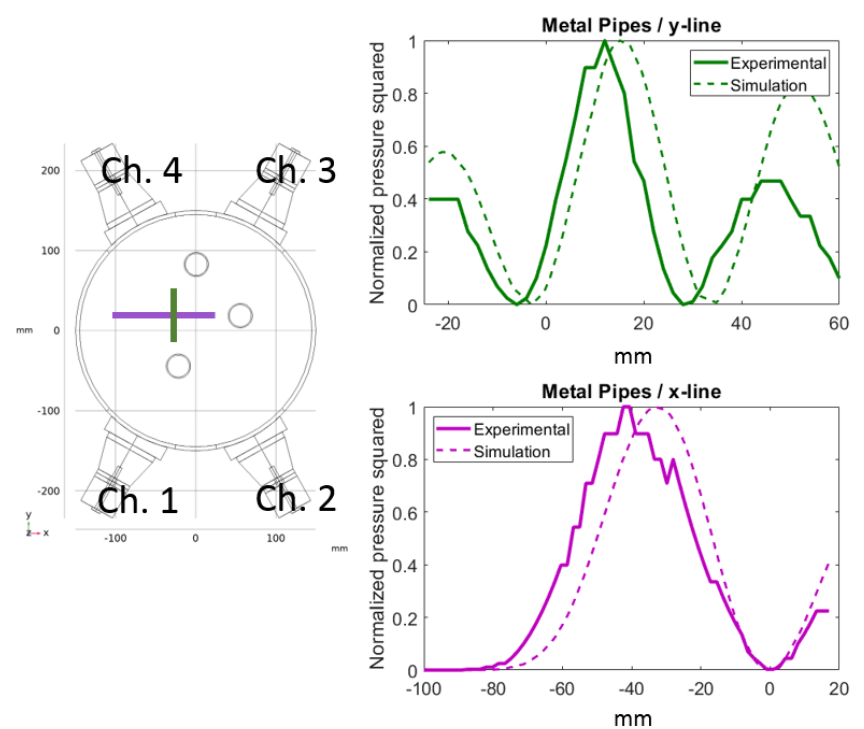

Fig. 6. Validation of the simulation with three additional pipes. Colored lines in the picture (left) show the line scans. The focal point is at the intersection of lines. Graphs show comparisons of experimental measurement (solid line) and simulation (dashed line).

\section{CONCLUSION}

We demonstrated that one can simulate forward-propagated signals in FEM, by generating an accurate model of the real-life setup, in which an excitation is simulated at the predefined location. We also demonstrated that accurate TR focusing can be achieved using signals generated in a simulation.

The results indicate that this method works both when focusing into homogenous media and when additional objects are placed into the media. The simulation results show that TR focusing can be achieved accurately in both studied cases. Experimental measurements agree with simulations, thus confirming the validity of simulation results.

\section{REFERENCES}

[1] M. Fink, C. Prada, F. Wu, D. Cassereau, Self Focusing in Inhomogeneous Media With "Time Reversal" Acoustic Mirrors, Proc. IEEE Ultrason. Symp., vol. 2, pp 681-686, 1989

[2] D. Cassereau, M. Fink, "Time reversal of ultrasonic fields - Part III: Theory af the Closed Time-Reversal Cavity", IEEE Trans. Ultrason. Ferroelec. Freq. Contr., vol 39(5), pp. 579-592, 1992.

[3] F. Wu, J.-L. Thomas, M. Fink, "Time reversal of ultrasonic fields - Part II: Experimental Results", IEEE Trans. Ultrason. Ferroelec. Freq. Contr., vol 39(5), pp. 567-578, 1992.

[4] C. Larmat, J.-P. Montagner, M. Fink, Y. Capdeville, A. Tourin, E. Clévédé, Time-reversal imaging of seismic sources and application to the great Sumatra earthquake, Geophysical Research Letters, vol. 33, 2006.

[5] R. Zhu, G. L. Huang, F. G. Yuan, Fast damage imaging using the timereversal technique in the frequency-wavenumber domain, Smart Mater. Struct, vol. 22, 2013.

[6] A. Sutin, H. Salloum, Prospective medical application of nonlinear time reversal acoustics, Proceedings of Meetings on Acoustics (Acoust. Soc. Am.), vol 34, 2018.

[7] J. Kober, Z. Dvorakova, Z. Prevorovsky, J. Krofta, Time reversal transfer: Exploring the robustness of time reversed acoutics in media with geometry perturbations, J. Acoust. Soc. Am., vol. 138, 2015.

[8] S. Yon, M. Tanter, M. Fink, Sound focusing in rooms: The time-reversal approach, J. Acoust. Soc. Am., vol. 113(3), 2003. 
[9] V. R. N. Santos, F. L. Teixeira, Application of time-reversal-based processing techniques to enhance detection of GPR targets, Journal of Applied Geophysics, vol. 146, 2016. 